
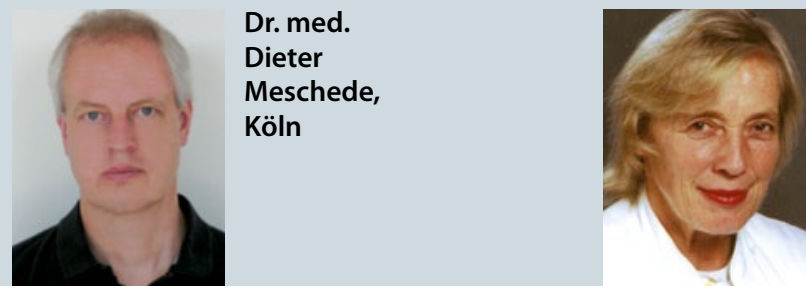

Prof. Dr. med.

Lieselotte

Mettler,

Kiel
Prof. Dr. med.

Tino F.

Schwarz,

Würzburg

\section{Fortgeschrittenes Endometriumkarzinom: Warum wirkt Chemo besser als Radiatio?}

\author{
Hat der Krebs das zervikale Stroma erfasst, verdüstert sich die Prognose von \\ Patientinnen mit fortgeschrittenem Endometriumkarzinom. Mit \\ Chemotherapie ist den meisten dieser Frauen besser geholfen als mit \\ abdomineller Radiatio. Weshalb, bleibt unklar.
}

$D^{i}$ ie Gynecologic Oncology Group(GOG)-122-Studie hatte 2006 ergeben, dass fünf Jahre nach einer Chemotherapie mit Doxorubicin plus Cisplatin noch 55\% der Patientinnen mit fortgeschrittenem Endometriumkarzinom (FIGO-Stadien III und IV) am Leben sind. Von den Patientinnen, die in der gleichen Situation eine Bestrahlung des gesamten Abdomens erhalten hatten, lebten noch $42 \%$ - ein signifikant schlechteres Ergebnis. Eine Gruppe US-amerikanischer Onkologen hat nun versucht herauszufinden, ob die Überlegenheit der Chemotherapie an bestimmte pathologische Merkmale der Tumorerkrankung geknüpft ist. $\mathrm{Zu}$ diesem Zweck haben sie die GOG-122Daten noch einmal retrospektiv hinsichtlich des progressionsfreien und des Gesamtüberlebens analysiert.

In die Ergebnisse gingen die Angaben zu 396 Patientinnen ein: 202 hatten eine Radiatio, 194 eine Chemotherapie erhalten. War der Tumor ins zervikale Stroma eingebrochen, erhöhte dies das Progressions- bzw. Sterberisiko während der Nachbeobachtungszeit (im Median 74 Monate) um $44 \%$. Für den Befall der Lymphknoten ergab sich ein Trend hin zu einer 7\%igen Erhöhung des Progressions-/Sterberisikos pro positivem Knoten.

Für den lymphovaskulären Befall und den Tumoreinbruch ins zervikale Stroma errechnete sich sowohl für das progressionsfreie als auch für das Gesamtüberleben ein Trend zugunsten eines Nutzens der Chemotherapie mit Doxorubicin und Cisplatin. Die Größenordnung des Vorteils im Vergleich zur Bestrahlung lag zwischen 5\% und 25\%. Doch die Konfidenzintervalle der Hazard Ratios schlossen den Wert 1 ein. Es ist damit statistisch gesehen nicht ausreichend wahrscheinlich, dass die berechneten Werte einer tatsächlichen Differenz entsprechen.

Die vorliegende retrospektive Aufarbeitung der GOG-122-Studie unterstützt zwar die bereits seit 2006 bekannten Ergebnisse. Sie liefert aber keine Belege für die Hypothese, wonach die Vorteile der Chemo- gegenüber der Strahlentherapie auf Faktoren der Tumorpathologie zurückgehen würden, als da wären: Befall des lymphovaskulären Raums, des zervikalen Stromas oder der pelvinen Lymphknoten.

Dr. Robert Bublak

Tewari KS et al. Association of number of positive nodes and cervical stroma invasion with outcome of advanced endometrial cancer treated with chemotherapy or whole abdominal irradiation: A Gynecologic Oncology Group study. Gynecol Oncol 2012; 125: 8793

Kommentar: Die GOG-122 Studie, die erstmalig 2006 die Überlegenheit einer adjuvanten Chemotherapie gegenüber einer Bestrahlung beim fortgeschrittenen Endometriumkarzinom dokumentierte, wies eine Reihe von Schwächen auf, die vielfach dis- kutiert wurden. So handelte es sich entgegen der primären Absicht nicht um eine reine Adjuvanzstudie, da $16 \%$ aller Frauen residuale Tumoren auch nach OP mit einer Größe von über $2 \mathrm{~cm}$ aufwiesen. Erhebliche Kritik kam auch von Seiten der Strahlentherapeuten, da im Rahmen dieser Studie eine in der adjuvanten Situation unübliche Bestrahlung in Form einer alleinigen Ganzabdomenbestrahlung mit $30 \mathrm{~Gy}$ und einem Boost im kleinen Becken von 15 Gy erfolgte. Kritisiert wurde ferner die hohe Toxizität der Studie, die bei $17 \%$ zu einem Studienabbruch führte. Es sollte mit der hier vorgestellten Re-Evaluation der Studie überprüft werden, ob mithilfe der Parameter Lymph- oder Hämangiosis, Befall des Zervikalstromas und der Zahl der befallenen Lymphknoten ein Risikokollektiv definiert werden kann, das in besonderem Maße von einer adjuvanten Chemotherapie profitiert.

Die prognostische Bedeutung dieser $\mathrm{Pa}-$ rameter konnte im Rahmen dieser Re-Evaluation nochmals bestätigt werden und es wurde ergänzend gezeigt, dass Patienten mit zervikaler Stromainvasion in besonderem Maße von einer adjuvanten Chemotherapie profitieren.

Diese Ergebnisse sind mittlerweile durch die vorgelegte Meta-Analyse zur Wertigkeit der adjuvanten Chemotherapie beim Endometriumkarzinom überholt worden. In dieser aktuellen Meta-Analyse wird die in den Leitlinien bereits festgelegte Vorgehensweise nun nochmals bestätigt: Patienten mit fortgeschrittenem Endometriumkarzinom, insbesondere mit Lymphknotenmetastasen, profitieren von einer adjuvanten Chemotherapie mit Platin und Taxol. Die optimale Behandlung in dieser Situation besteht offenbar in der Kombination einer adjuvanten Chemotherapie mit einer Bestrahlung.

Prof. Dr. Peter Mallmann 